\title{
BMJ Open Is smoking a risk factor for tinnitus? A systematic review, meta-analysis and estimation of the population attributable risk in Germany
}

Annette Veile, ${ }^{1}$ Heiko Zimmermann, ${ }^{1}$ Eva Lorenz, ${ }^{1,2}$ Heiko Becher ${ }^{1,3}$

To cite: Veile A, Zimmermann $\mathrm{H}$, Lorenz $\mathrm{E}$, et al. Is smoking a risk factor for tinnitus? A systematic review, metaanalysis and estimation of the population attributable risk in Germany. BMJ Open 2018;8:e016589. doi:10.1136/ bmjopen-2017-016589

- Prepublication history and additional material for this paper are available online. To view these files, please visit the journal online (http://dx.doi. org/10.1136/bmjopen-2017016589).

Received 23 February 2017 Revised 24 November 2017 Accepted 27 November 2017
Check for updates

${ }^{1}$ Institute of Public Health, Unit of Epidemiology and Biostatistics, University of Heidelberg, Heidelberg, Germany

${ }^{2}$ Institute of Medical

Biostatistics, Epidemiology and Informatics, University Medical Center Mainz, Mainz, Germany ${ }^{3}$ Institute of Medical Biometry and Epidemiology, University Medical Center HamburgEppendorf, Hamburg, Germany

Correspondence to Professor Heiko Becher; h.becher@uke.de

\section{ABSTRACT}

Objective To assess the epidemiological association of smoking status and tinnitus with a systematic review and meta-analysis and to estimate the population attributable risk in Germany.

Data sources A systematic literature search in PubMed and ISI-Web of Science Core Collection resulted in 1026 articles that were indexed until 15 September 2015. Additionally, proceedings of the international tinnitus seminars and reference lists of relevant articles were screened.

Study selection Two reviewers searched independently for epidemiological studies. Tinnitus as a manifestation of tumours, vascular malformations, specific syndromes or as a consequence of surgical and medical treatment was not considered. Moreover, studies conducted among patients of ear, nose and throat clinics were excluded.

Data extraction If only raw data were provided, effect sizes were calculated. Further unpublished data were received by corresponding authors.

Data synthesis Data of 20 studies were pooled. Current smoking (OR 1.21, 95\% $\mathrm{Cl} 1.09$ to 1.35), former smoking (OR $1.13,95 \% \mathrm{Cl} 1.01$ to 1.26$)$ and ever smoking (OR 1.20, $95 \% \mathrm{Cl} 1.11$ to 1.30 ) were significantly associated with tinnitus. Moreover, sensitivity analyses for severe tinnitus (OR $1.32,95 \% \mathrm{Cl} 1.10$ to 1.58 ) and for studies of superior quality (OR $1.15,95 \% \mathrm{Cl} 1.03$ to 1.29 ) showed increased risks. According to this, the population attributable risk estimate in Germany is $3.5 \%$.

Conclusion There is sufficient evidence that smoking is associated with tinnitus. As the review mainly consists of cross-sectional studies, the observed correlation does not give evidence of a causal relationship. Due to the impact of various confounders, further research is needed to provide more evidence on the strength of association and causal relationships.

\section{INTRODUCTION}

Tinnitus is defined as the perception of a sound in absence of an acoustic sound source. ${ }^{1}$ Up to $68.5 \%$ of the population perceive tinnitus as a temporary condition at least once during lifetime,${ }^{2}$ notably occurring after extensive noise exposure or during stressful life episodes. However, depending
Strengths and limitations of this study

- Results of 20 studies among different ethnicities and age patterns have been pooled.

- All eight subgroup analyses showed a significant effect of smoking on tinnitus, except for two.

- Multiple sources of bias may have influenced the results to some degree.

- Substantial heterogeneity such as different definitions of tinnitus makes comparisons of studies difficult.

- Recently published studies could alter the results of the meta-analysis. However, in a rough update of the search on 1 September 2016, no articles were detected that provided additional data to the analysis.

on loudness, persistence and individual coping skills it can reach a pathologic and extremely distressing extent. In some cases, tinnitus entails hearing problems because of an impaired acoustic discrimination. Severe tinnitus affects the quality of life by causing sleeping problems, depression, social withdrawal, work incapacity and cognitive impairment. ${ }^{3-5}$ The prevalence of severe tinnitus varies from $1.5 \%$ to $6.9 \%$ depending on the definition applied. ${ }^{6}$ So far, no agreed upon definition has been established to differentiate between a trivial and a pathologic form of tinnitus. In recent decades, various pathophysiological theories have been proposed to explain tinnitus formation in different sections of the auditory pathway or central regions. ${ }^{8}{ }^{9}$ However, no model has been generally approved, most probably reflecting multiple or multifactorial aetiologies of tinnitus. One widely held hypothesis of tinnitus formation is the dysfunction of outer and inner hair cells. Beside noise exposure, hypoxia and ischaemia are discussed as major triggering factors in the dysfunction of these cells. ${ }^{10}$ Smoking-mediated vascular 
dysfunction and arteriosclerosis could be crucial factors in the existence of ischaemic or hypoxic conditions. Research showed that nicotine and other constituents of cigarette smoke promote vasoconstriction, aggregation of platelets, increased blood viscosity and haemostasis by interfering with the synthesis of prostacycline, thromboxane and fibrinogen. ${ }^{1-14}$ Moreover, local hypoxia may be exacerbated by higher levels of carboxyhaemoglobin in smokers compared with non-smokers. ${ }^{15}$ Besides, ototoxic agents such as hydrogen cyanide, lead, styrene and toluene can be found among the constituents of cigarette smoke. ${ }^{16}{ }^{17}$ The formation of a hearing impression is submitted to a complex neuronal network of reinforcing and inhibiting influences, whose homeostasis is highly susceptible to minor changes. Nicotine influences the transmission of acoustic information ${ }^{18}$ by interfering with neurotransmitter release in different sections of the central nervous system. ${ }^{19} 20$ Most notably, synaptic plasticity, which is recently discussed in the context of tinnitus genesis, is altered by nicotine. The effects of nicotine are highly diverse and depend on sections of the central nervous system, concentration and duration of administration. ${ }^{19}{ }^{20}$ For the above-outlined mechanisms, smoking is suspected as a biologically plausible risk factor in the formation of tinnitus. ${ }^{721}$ Stouffer and Tyler asked tinnitus patients about their subjective theories of tinnitus aetiology. Most of them attributed tinnitus to noise, hearing loss or other diseases, but none of them suspected tinnitus as result of smoking. ${ }^{22}$ According to the various pathophysiological theories, an abundant number of therapies have been proposed to treat tinnitus in causal approaches. Yet, none of these therapies is endorsed in the current guidelines, ${ }^{2324}$ which is either due to unfavourable side-effect profiles or the lack of evidence-based efficacy. Merely symptomatic therapies such as cognitive behavioural therapy or sound therapy for the improvement of coping with tinnitus are recommended. ${ }^{23}{ }^{24}$ The unsatisfactory range of evidence-based therapeutic options supports the need for the prevention of tinnitus and research on its risk factors. To investigate the hypothesis of smoking as a risk factor, we conducted a systematic review and meta-analysis of epidemiological studies. This review is oriented towards the recommendations of Preferred Reporting Items for Systematic Reviews and Meta-Analyses (PRISMA) Statement, ${ }^{25}$ Meta-analysis Of Observational Studies in Epidemiology (MOOSE) Statement ${ }^{26}$ and Cochrane Handbook for Systematic Reviews of Interventions. ${ }^{27}$

\section{METHODS}

\section{Search strategy and selection process}

The databases searched were PubMed and ISI-Web of Science Core Collection. All articles indexed until 15 September 2015 with a language restriction to English, French and German were taken into account. Boolean Operators were used for assembling search strings by combining Medical Subject Headings (MeSH) terms and text words (tw) with explosion terms in PubMed and topic field tags (TS) in ISI-Web of Science, respectively. The precise search strategy in PubMed is shown in figure 1. The search strategy used in ISI-Web of Science is available from the corresponding author on request.

Two reviewers (AV and HZ) independently screened titles and abstracts for eligibility. In case of discordance, the question of inclusion was resolved by a discussion with a third reviewer (HB). Further studies were retrieved as references in relevant articles. In addition, proceedings of the international tinnitus seminars were screened for relevant articles. ${ }^{28-30}$ On 1 September 2016, a crude update of the search was performed. To the best of our knowledge, there were no articles published before this date which provided additional data to the analysis.

\section{Criteria for inclusion and exclusion}

All studies corresponding to an epidemiological study design that provided effect sizes or other data for the association of smoking as exposure and tinnitus as outcome were included. There was no restriction to study population by age or ethnic background. Some articles did not

\begin{tabular}{|ll|}
\hline$\# 1$ & Search ((("smoking"[MeSH Terms]) OR "risk factors"[MeSH Terms]) OR "tobacco"[MeSH Terms]) \\
& OR "tobacco products"[MeSH Terms]) OR "tobacco use disorder"[MeSH Terms] \\
$\# 2$ & Search ("tinnitus"[MeSH Terms]) AND \#1 \\
$\# 3$ & Search smok*[Text Word] \\
$\# 4$ & Search cigar*Text Word] \\
$\# 5$ & Search tobac*[Text Word] \\
$\# 6$ & Search risk factor*[Text Word] \\
$\# 7$ & Search associat* factor*[Text Word] \\
$\# 8$ & Search correlat" factor*Text Word] \\
$\# 9$ & Search (((((\#8) OR \#7) OR \#6) OR \#5) OR \#4) OR \#3 \\
$\# 10$ & Search (tinnitus[Text Word]) AND \#9 \\
$\# 11$ & Search (\#10) OR \#2
\end{tabular}

Figure 1 Search strategy applied in the database PubMed. In PubMed Boolean Operators ('OR' and 'AND') were used for assembling the search string by combining Medical Subject Headings (MeSH) terms and text words (tw) with explosion terms (marked by '*'). 
provide effect sizes, but raw data that allowed to calculate these. In these cases, Review Manager V.5.2 software was used to calculate the ORs.

Studies dealing with objective tinnitus, for example due to cerebrovascular malformations, or with tinnitus as a direct consequence of tumours, surgical or medical treatment, particular syndromes such as Menière's disease or Conan syndrome, etc were also left out. Equally, studies conducted among patients of ear, nose and throat (ENT) clinics were excluded.

The diagnose spectre of ENT clinics covers to a great extent diseases which are triggered by active or passive smoking. Some of these are for instance sinusitis, ${ }^{31} 32$ oropharyngeal cancer, ${ }^{33} 34$ otitis media ${ }^{35} 36$ and hearing impairment. ${ }^{37}$ Thus, we suspect the smoking prevalence of this reference group to be higher than the smoking prevalence of the common population that does not suffer from tinnitus. As this would have underestimated the association of smoking and tinnitus, we excluded studies which were conducted in ENT clinics.

\section{Assessment of study quality, risk of bias and comparability}

In accordance to MOOSE Statement, sensitivity analyses were performed for current smoking instead of giving weight to studies depending on the result of a certain quality score. ${ }^{26}$ These quality scores are considered controversial, which is on one hand due to the lack of validity and on the other hand due to the fact that methodological assets or flaws can hardly be quantified by a certain value. ${ }^{27} 38$ In order to rate and select studies for a sensitivity analysis of superior quality, we specially designed a tool for this review. In a synopsis of several established quality assessment tools, ${ }^{39-42}$ single items that deemed suitable and essential to this topic were retrieved. The selected items concerned susceptibility to bias, the quality of reporting as well as comparability with other studies.

\section{Data extraction and statistical analysis}

The characteristics and effect sizes of included studies were extracted and presented in a spreadsheet. If only tabulated data were provided, the corresponding odds ratios (OR) were calculated if possible. Corresponding authors were contacted to ask for precise effect sizes if the results were described as 'not significant' without providing further evidence.

Data extraction and calculation of effect sizes were performed by one reviewer twice with an interval of several months to avoid a recall. Moreover, the extracted and calculated data were double-checked by another coauthor (HB). To objectify the probability of publication bias, funnel plots were checked for a symmetrical distribution. The analyses and calculations were done with Review Manager V.5.3. The calculation of the standard error (SE) from the $95 \%$ confidence interval (CI) was done with excel. To account for the heterogeneity of the studies, the summary measures were estimated with a random-effects model. Effect sizes were entered in the analysis programme in the format of $\log \mathrm{OR}$ and SE. The measures to describe the heterogeneity are $\mathrm{Chi}^{2}\left(\mathrm{X}^{2}\right)$ representing the observed variation, and the degrees of freedom (df) indicating the expected variation or heterogeneity. ${ }^{43} \mathrm{I}^{2}$ indicates the proportion of the observed heterogeneity which is due to true heterogeneity. ${ }^{27}$ In contrast to $\mathrm{I}^{2}$ as a relative indicator, $\mathrm{T}^{2}$ represents the absolute amount of heterogeneity. ${ }^{43}$

Some studies provided effect sizes separately by gender, ${ }^{44}$ years since smoking cessation ${ }^{45} 46$ or other features. ${ }^{77} 48$ If two or more effect sizes of one study were entered into the Review Manager V.5.2 software, they would have been computed as separate studies. As the expected variation correlates with the number of studies (df), this would have over-rated the expected variation. Therefore, the effect sizes of these studies were pooled to one summary estimate in a fixed-effect model. One study showed ORs for never smoking with current smoking as a reference group. ${ }^{49}$ In this case, the effect sizes were inversed. In another case, ${ }^{7}$ the ratio of two effect sizes had to be calculated to isolate the association between smoking and tinnitus, which was done orienting towards a method by Altman and Bland. ${ }^{50}$ One single article provided estimates as $\mathrm{HR}^{46}$ and another as prevalence ratios (PR). ${ }^{7}$ These effect sizes were treated equally to ORs, which is methodologically not accurate. However, as the disease prevalence was low and the estimates close to a value of 1 , these values were considered as comparable to ORs.

Given that incapacitating and frequent tinnitus might be a completely different pathophysiological entity than transient non-bothersome tinnitus, not differentiating between these could attenuate the real effect of smoking. Therefore, a second sensitivity analysis was performed for studies examining severe tinnitus. Severe tinnitus was defined either as 'very' or 'extremely annoying', 'bothersome', occurring often up to continuously or as tinnitus considered as 'a big problem'.

\section{RESULTS}

After removal of duplicates, literature research yielded 1026 articles. Some duplicate articles differed in minor features such as publication date, journal, etc and were treated as two articles in order to facilitate the finding of the full text. Additional 172 articles were retrieved in the reference list of relevant articles later on and are already included in the following numbers. After excluding 608 articles based on the title, and other 389 articles based on abstract, 201 full-text articles were searched for relevant data. All in all, 35 articles investigating the association between smoking status and tinnitus were detected. Of those, five articles were double publications ${ }^{51-55}$ and three articles were excluded as they were conducted among patients of ENT clinics. ${ }^{56-58}$ One study was excluded because the selection of study participants was apparently biased. ${ }^{59}$ Another observational study by Martinez et $a b^{60}$ showed strong evidence of bias as the available data on smoking status differed widely between subjects with 


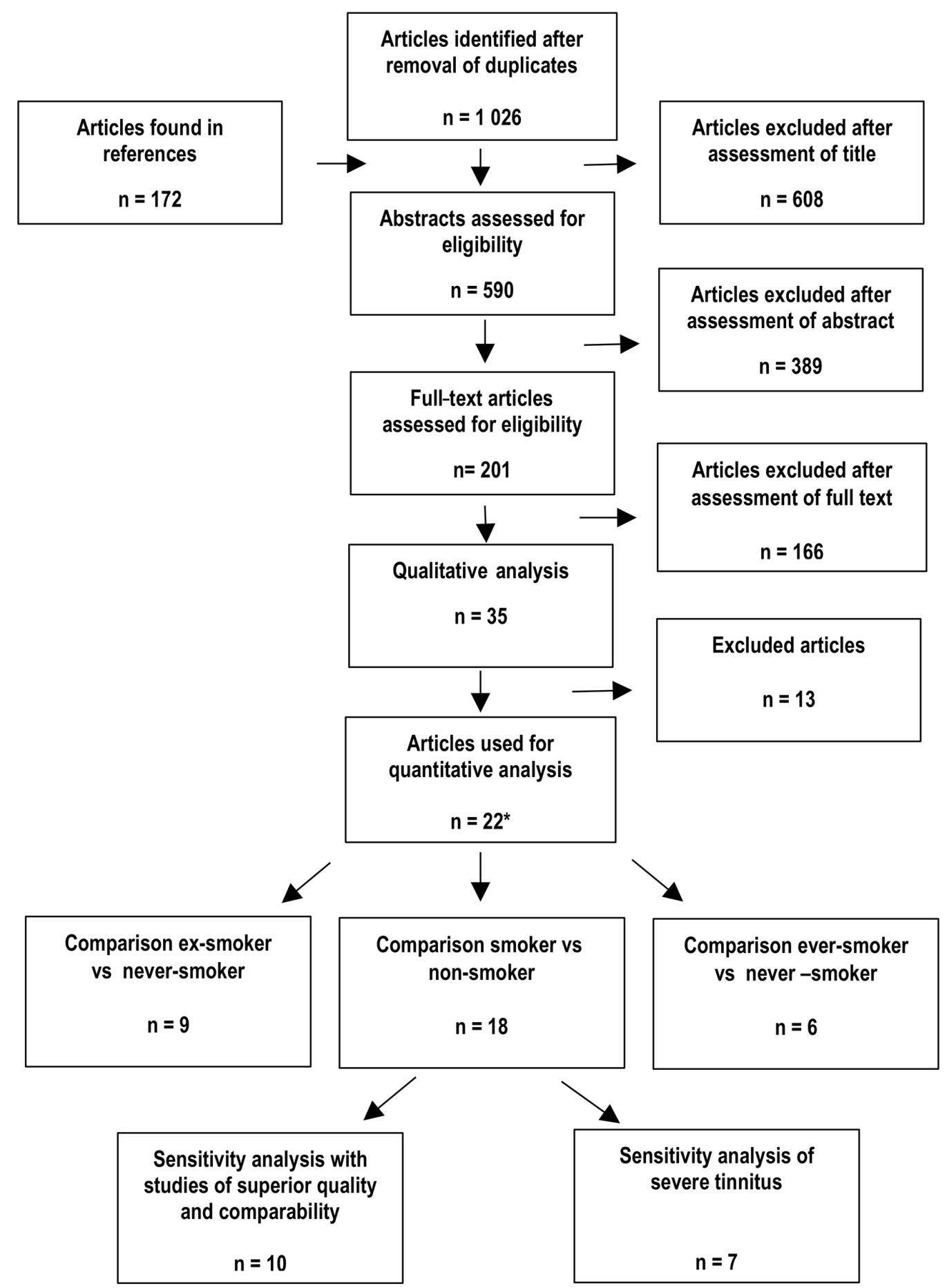

Figure 2 Flow diagram of study selection. The flow chart shows the number of articles that were screened and excluded in different steps of the selection progress. *Due to double publications the 22 articles cover 20 different studies.

and without tinnitus. In another study by Paschoal $e t a l^{61}$ the selection of the study group and the assessment of tinnitus is inadequately described. All these studies were therefore not considered further. As four articles did not provide effect sizes for non-significant results, we contacted the authors. As a result, two of these articles could be included. After having excluded 13 articles for the above-mentioned reasons, 22 remained eligible for quantitative analyses. Among these, one article presented results of two studies, ${ }^{21}$ while the following studies were published twice.

Palmer $e t a l^{7}$ assessed the risk in terms of current, former or never-smokers and in another article in terms of ever or never smoking. ${ }^{62}$ Both articles could be used across the different comparisons. Data of the Blue Mountains Hearing Study were once analysed in terms of general tinnitus, ${ }^{63}$ whereas another article focused on severe tinnitus. ${ }^{64}$ In the sensitivity analysis for severe tinnitus, the 


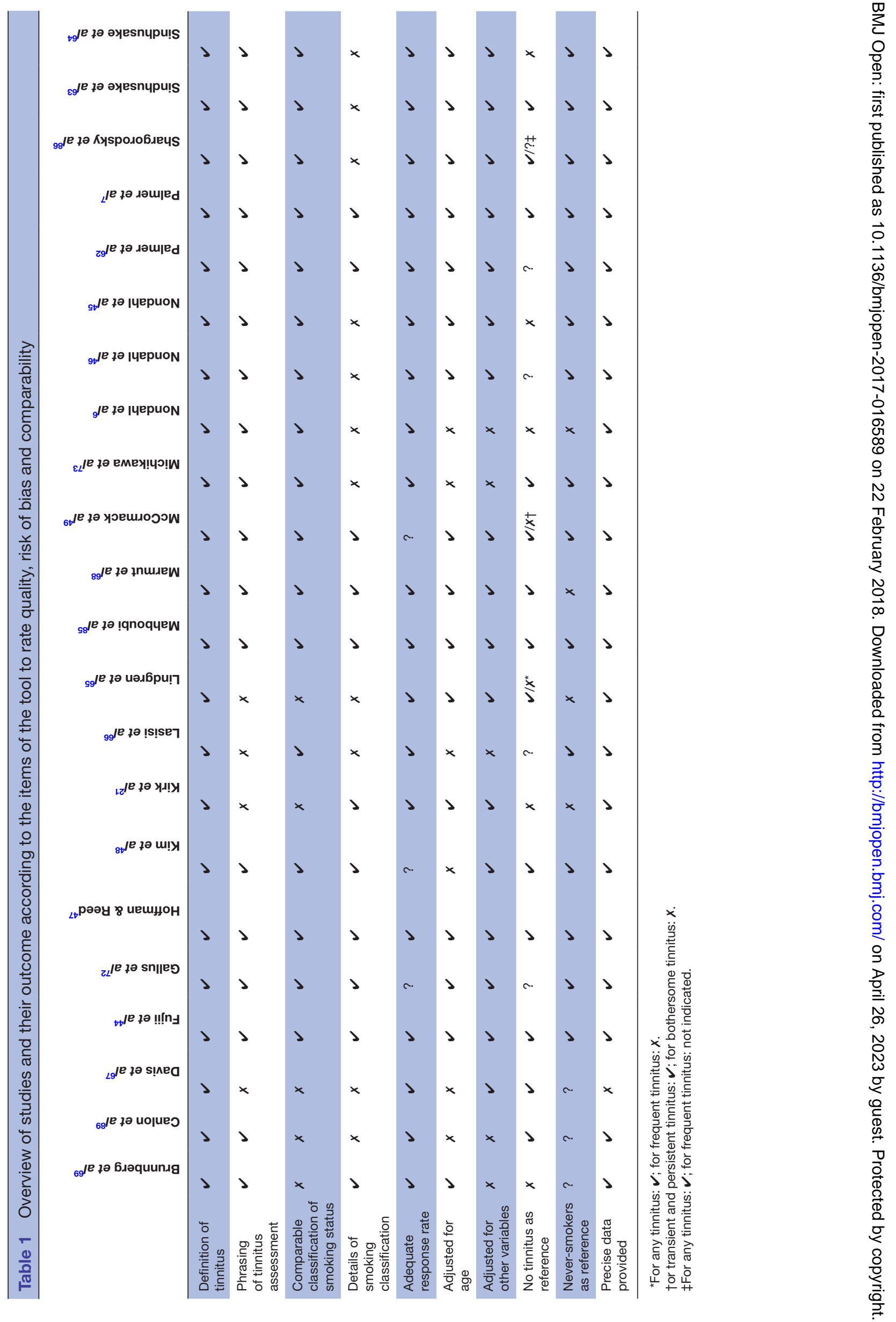




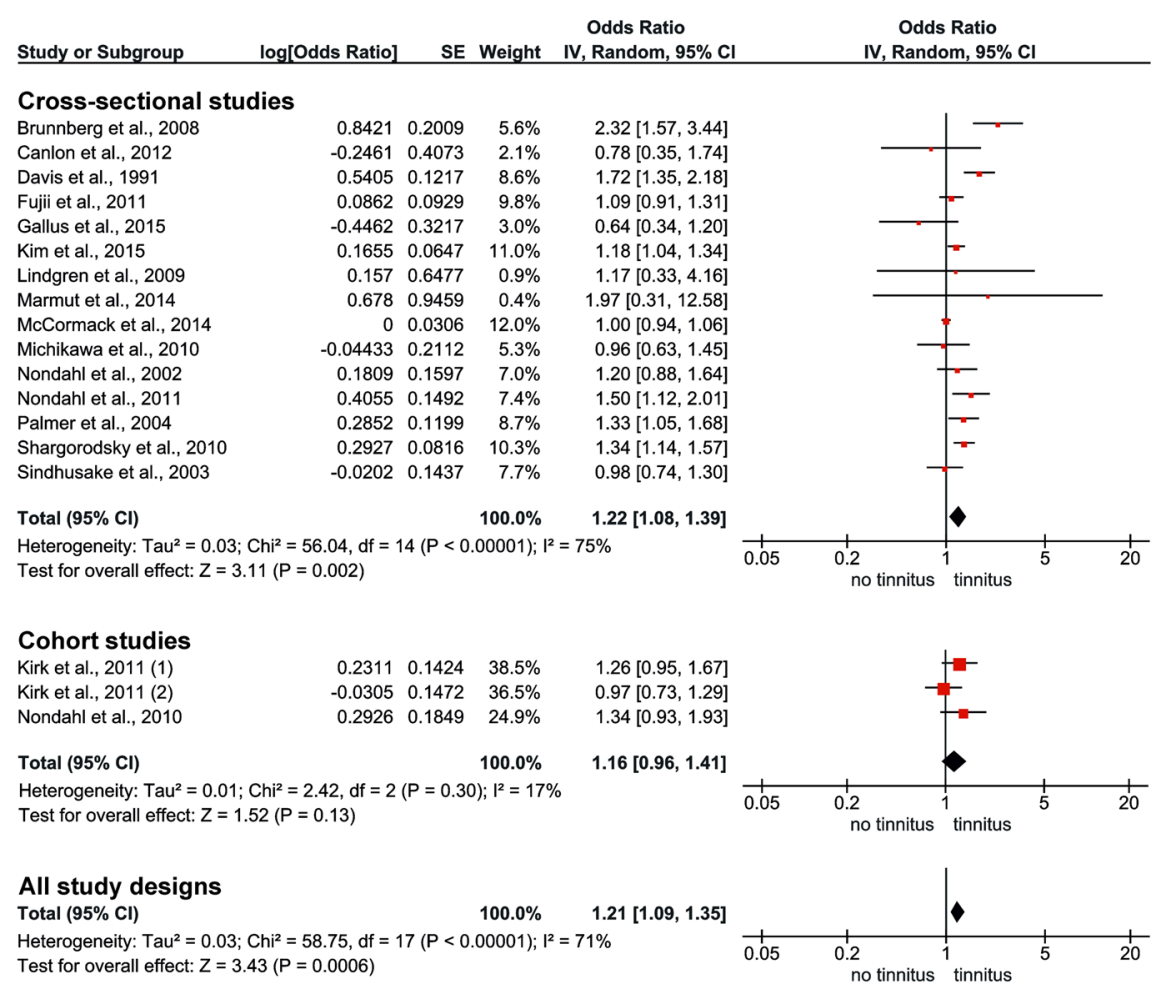

Figure 3 Meta-analysis of tinnitus in current smokers. Studies were combined using a random effects generic inverse variance model. The first two blocks show forest plots of cross-sectional and cohort studies separately. The effect size of each study is illustrated on a logarithmic scale by a red square, whose surface is proportional to the weight of the study. ORs $>1$ show a positive association of smoking on tinnitus. The horizontal bars represent the $95 \% \mathrm{Cl}$. Summary estimates are represented by a black diamond. The third diamond illustrates the summary estimate of the comparison with all study designs. The ' $P$ ' value refers to the test of heterogeneity. This means that a statistical significant $P$ value indicates heterogeneity, whereas a nonsignificant value does not rule out heterogeneity.

effect sizes for severe tinnitus were included. In all other analyses, the associations for general tinnitus were used. From the Epidemiology of Hearing Study, there was one article about the cross-sectional analysis at the beginning of the study period ${ }^{6}$ and another one about the 10-year incidence. ${ }^{45}$ In the final analysis, both assessments were included as separate studies.

As these double publications provided important data for subgroup analyses, the articles were not discarded. Due to this, the 22 articles represent 20 studies. An overview of the selection process is described in figure 2. Online supplementary table shows relevant data of the included articles, such as year of publication, country, study design, sample size, tinnitus and smoking definitions. The results of the assessment of quality, risk of bias and comparability are provided in table 1 .

The tool to rate quality, risk of bias and comparability comprises 10 items that are described below:

\section{Definition of tinnitus provided}

This item was fulfilled if any crude definition of tinnitus was provided.

\section{Phrasing of tinnitus assessment provided}

This item was considered fulfilled if the exact phrasing of tinnitus assessment of the questionnaires or interviews was cited. If the definition was given indirectly the item was rated as not fulfilled. This was the case in three studies. ${ }^{216566}$ One article did not give any evidence of the phrasing. ${ }^{67}$

\section{Comparable classification of smoking status}

The classification of smoking in terms of current, former and never-smokers or ever-smokers and neversmokers was provided in the most of the studies and thus considered as comparable. If smoking status was assessed as a dichotomous variable, it was not clear whether former smokers were assigned to the smoking or non-smoking group or excluded from the analysis. Thus, in these cases, the item was judged as not fulfilled. Some studies provided effect sizes stratified by the years 'since smoking cessation' or the years, in that participants 'smoked daily'. These effect sizes were pooled to one summary estimate for former smokers and ever-smokers, respectively. In that way, such smoking classifications were declared as comparable, too.

\section{Details of smoking classification provided}

Details of smoking classification were, for instance, the amount or regularity of consumption that defined a certain smoking status. Other examples were the time of abstinence, which was used to distinguish current from 


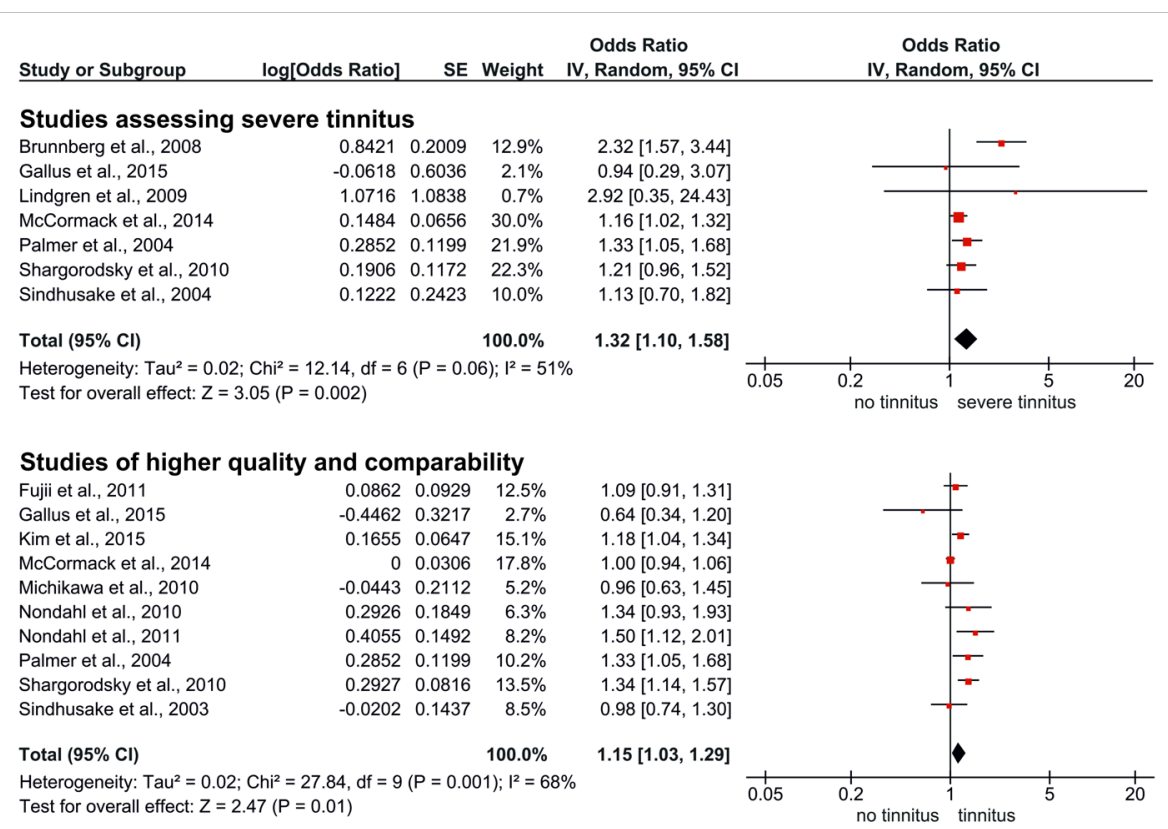

Figure 4 Sensitivity analyses of current smoking and tinnitus. Studies were combined using a random effects generic inverse variance model. The first block shows the forest plot of a sensitivity analysis pooling studies on severe tinnitus. The second block summarises studies of higher quality and comparability. The effect size of each study is illustrated on a logarithmic scale by a red square, whose surface is proportional to the weight of the study. ORs $>1$ show a positive association of smoking on tinnitus. The horizontal bars represent the $95 \% \mathrm{Cl}$. Summary estimates are represented by a black diamond. The ' $\mathrm{P}$ ' value refers to the test of heterogeneity. This means that a statistical significant $\mathrm{P}$ value indicates heterogeneity, whereas a non-significant value does not rule out heterogeneity.

former smokers. This criterion was equally regarded as fulfilled if the phrasing of smoking assessment was cited.

\section{Adequate response rate}

An adequate response rate was set as $\geq 40 \%$.

\section{Adjusted for age}

This item was considered fulfilled if the results were controlled for age. Studies conducted among the same age group were treated as age-adjusted. ${ }^{68} 69$

\section{Adjusted for other variables}

Factors considered in the adjustments of effect sizes varied widely. The criterion was considered fulfilled if there was an adjustment for at least one other factor than age.

\section{No tinnitus as reference}

In some studies, the reference group comprised subjects with moderate or rare tinnitus. In this case, the item was judged as not fulfilled.

\section{Never-smokers as reference}

The item was considered fulfilled if the reference category consisted exclusively of never-smokers. One study provided estimates for never smoking with current smoking as reference. ${ }^{49}$ In this case, we inverted the OR and assessed the item as fulfilled.

\section{Precise data provided}

Precise results were defined as ratios with $95 \%$ CI, or data to calculate these. In contrast to those, one study provided a ratio without CI.
To select studies for a sensitivity analysis of superior quality and comparability, the following items were set as obligatory:

1. Definition of tinnitus provided;

2. Comparable classification of smoking status;

3. Never-smokers as a 'reference'.

The first analysis of current smoking (figure 3) showed an increased risk of tinnitus with an OR of 1.22 (95\% CI 1.08 to 1.39) for cross-sectional studies and an OR of 1.16 (95\% CI 0.96 to 1.41 ) for cohort studies, respectively. In an inclusion of all study designs, an OR of 1.21 (95\% CI 1.09 to 1.35 ) was observed. The $\mathrm{I}^{2}$ of $71 \%$, which reflects the proportion of true heterogeneity to observed heterogeneity is substantial, whereas $\mathrm{T}^{2}$ as an estimate of the variance of true effect sizes is 0.03 .

In the sensitivity analyses, significantly increased risks were indicated for severe tinnitus (OR 1.32, 95\% CI 1.10 to 1.58 ) and for studies of superior quality and comparability (OR 1.15, 95\% CI 1.03 to 1.29) (figure 4).

The results for former smoking are presented in figure 5 . In a first analysis of cross-sectional studies, the pooled OR was 1.09 (95\% CI 0.98 to 1.20$)$. Adding one cohort study to the analysis yielded a significantly increased risk with an OR of 1.13 (95\% CI 1.01 to 1.26). The $\mathrm{I}^{2}$ demonstrated substantial heterogeneity $(61 \%)$. However, $\mathrm{T}^{2}$ as an indicator of total heterogeneity was rather small compared with the other analyses $\left(\mathrm{T}^{2}=0.02\right)$. 


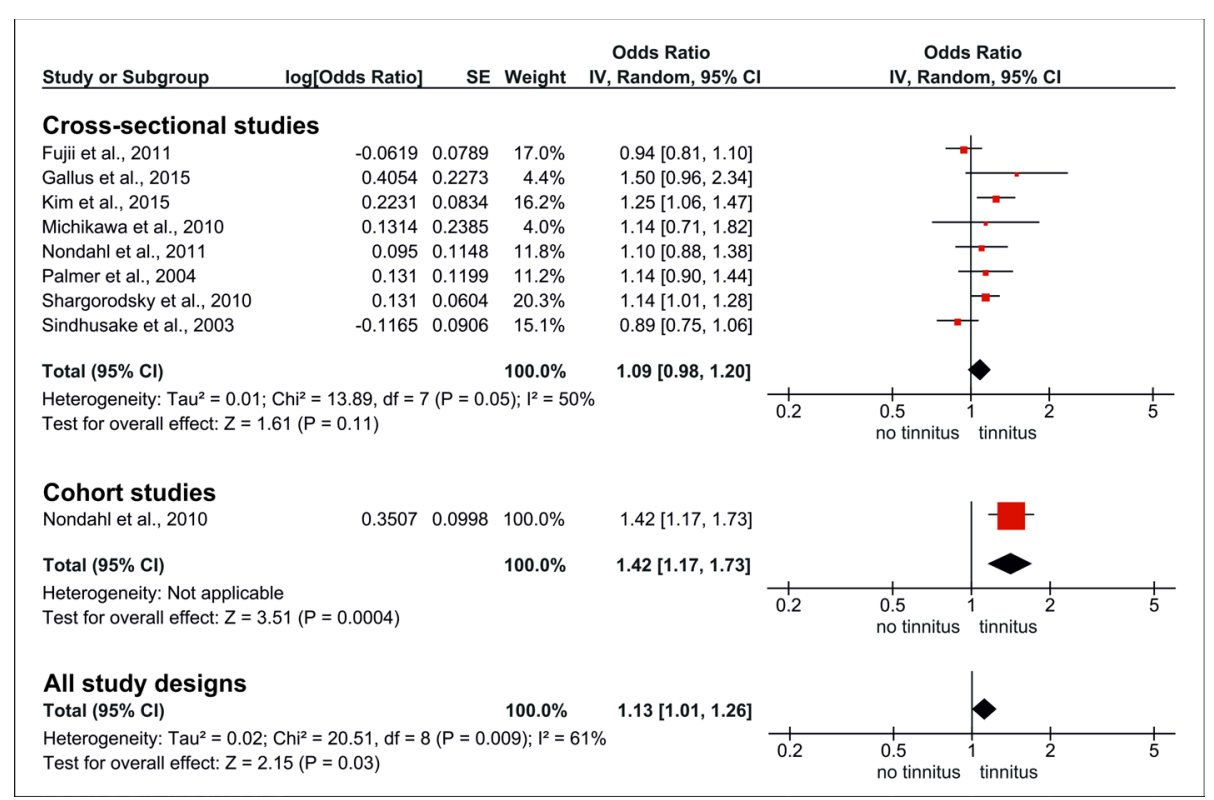

Figure 5 Meta-analysis of tinnitus in former smokers. Studies were combined using a random effects generic inverse variance model. The first two blocks show forest plots of cross-sectional and cohort studies separately. The effect size of each study is illustrated on a logarithmic scale by a red square, whose surface is proportional to the weight of the study. ORs $>1$ show a positive association of smoking on tinnitus. The horizontal bars represent the $95 \% \mathrm{Cl}$. Summary estimates are represented by a black diamond. The third diamond illustrates the summary estimate of the comparison with all study designs. The ' $P$ ' value refers to the test of heterogeneity. This means that a statistical significant $P$ value indicates heterogeneity, whereas a nonsignificant value does not rule out heterogeneity.

Ever smoking (figure 6) correlated significantly with tinnitus in cross-sectional studies (OR 1.17, 95\% CI 1.05 to 1.30 ) and cohort studies (OR 1.35, 95\% CI 1.11 to 1.64). In the analysis of all study designs, the OR observed was 1.20 (95\% CI 1.11 to 1.30 ). In this subgroup, heterogeneity was negligible $\left(\mathrm{T}^{2}=0.00, \mathrm{I}^{2}=20 \%\right)$.
The funnel plots of current smoking, former smoking and ever smoking illustrated a symmetrical distribution, thus providing no evidence of publication bias. The associated figures are not shown but are available from the corresponding author upon request.

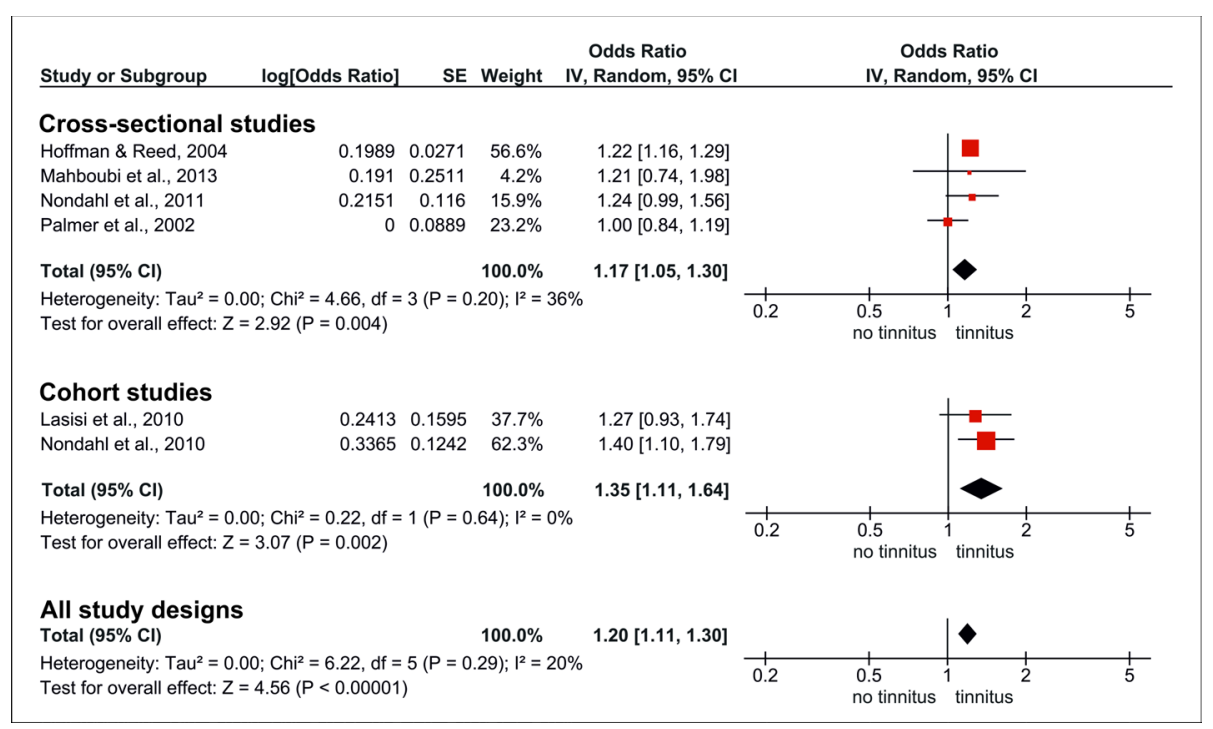

Figure 6 Meta-analysis of tinnitus in ever-smokers. Studies were combined using a random effects generic inverse variance model. The first two blocks show forest plots of cross-sectional and cohort studies separately. The effect size of each study is illustrated on a logarithmic scale by a red square, whose surface is proportional to the weight of the study. ORs $>1$ show a positive association of smoking on tinnitus. The horizontal bars represent the $95 \% \mathrm{Cl}$. Summary estimates are represented by a black diamond. The third diamond illustrates the summary estimate of the comparison with all study designs. The ' $P$ ' value refers to the test of heterogeneity. This means that a statistical significant $P$ value indicates heterogeneity, whereas a nonsignificant value does not rule out heterogeneity. 
The population attributable risk (PAR) indicates the proportion of a disease which can be attributed to a certain risk factor. ${ }^{70}$ For its calculation, the smoking prevalence of $24.5 \%$, assessed by the German Federal Statistical Office in $2013^{71}$ and the OR of the sensitivity analysis for superior quality and comparability (OR 1.15) was used. This resulted in a PAR of $3.5 \%$, which means that $3.5 \%$ of tinnitus cases are due to smoking if smoking is a causal factor of tinnitus. The PAR for severe tinnitus (OR 1.32 ) is $7.3 \%$.

\section{DISCUSSION}

In the final comparisons, which included all study designs as well as in the sensitivity analyses, smoking could be identified as a significant, although not very strong risk factor for the manifestation of tinnitus.

Considering smoking as a causal factor of tinnitus, it could be expected that the risk of current smokers is somewhat higher than the one of former smokers. We find a slight difference (OR 1.21 vs 1.13) in that direction. The risk of ever-smokers (OR 1.20) is almost identical to the risk of current smokers (OR 1.21).

A large variation in effect estimates between the included studies can either be explained by random error or by true heterogeneity of effects. In the analysis of current smoking, a substantial heterogeneity with an $\mathrm{I}^{2}$ of $71 \%$ and a $\mathrm{T}^{2}$ of 0.03 was observed. Thus, sensitivity analyses were performed to investigate this heterogeneity. The heterogeneity of the sensitivity analysis of severe tinnitus was slightly less pronounced $\left(I^{2}=51 \%, T^{2}=0.02\right)$. In the sensitivity analysis of superior quality and comparability, the relative heterogeneity was still substantial $\left(I^{2}=68 \%\right)$, but referring to a small amount of absolute heterogeneity $\left(\mathrm{T}^{2}=0.02\right)$. There are various factors that explain the observed heterogeneity. The definitions of tinnitus varied widely in severity and in terms of the period observed. ${ }^{447273}$ For instance, some studies assessed the life-time prevalence of tinnitus, whereas others assessed the prevalence of the preceding month. Similarly, distinct definitions of smoking behaviour were used. Among the study populations, there were large differences concerning age pattern, ethnicity, noise exposure, etc. Some of the studies controlled for risk factors such as noise exposure, age and hearing impairment, whereas other studies presented crude effect sizes.

\section{Possible confounding variables}

Smokers are known to differ from non-smokers in a range of health behaviours which promote the development of tinnitus. Literature shows that smokers are more likely to be exposed to occupational ${ }^{7}$ or leisure noise ${ }^{74}$ to reject the use of hearing protections devices ${ }^{75}$ and to exhibit behaviours predisposing to an increased cardiovascular morbidity. ${ }^{76}$ In addition, an association of tinnitus with anxiety and depressive disorders can be observed. ${ }^{778}$ The causal relationship is not clear, as tinnitus is possible as consequence, comorbidity or origin of depression. ${ }^{78}$ In turn, people suffering from depression or anxiety disorders seem to be more likely to be smokers, ${ }^{79} 80$ which could partly enhance the observed association. Other interfering variables are psychological stress or weak stress coping skills, which predispose to both tinnitus ${ }^{81} 82$ and smoking behaviour. ${ }^{83}$ Stress can also be a consequence of tinnitus and the urge to smoke regularly. In that way, it is even possible that stress due to tinnitus enhances smoking behaviour.

There is strong evidence that hearing impairment is associated with both smoking and tinnitus. ${ }^{37} 4547$ Thus, Chung et $a l^{84}$ suggested that the association of smoking and tinnitus might be indirect through the association with hearing impairment. However, studies adjusting for hearing impairment still showed positive correlations of smoking and tinnitus. ${ }^{46585}$ All of the above mentioned correlating variables are complexly intertwined, reflecting multicausal relationships. It is crucial to point out that studies adjusting for noise exposure ${ }^{747}$ stress $^{7}$ or cardiovascular factors ${ }^{86}$ nevertheless indicated a positive correlation between smoking status and tinnitus.

\section{Strengths and limitations}

Even though the visual assessment of funnel plots did not indicate a publication bias, there is evidence for the existence of outcome reporting bias. This kind of bias is present if non-significant results of examined associations within a study are reported only partly or not mentioned at all. ${ }^{87}$ There were two studies that could not be included, as they described the association of smoking and tinnitus solely as 'not significant' without providing effect sizes. ${ }^{848}$ The lacking inclusion of these two non-significant results enhanced the pooled effect estimate. But as these two studies represent $<1 \%$ of the total sample size of this analysis, the effect is negligible. However, further studies may exist which observed non-significant associations and did not report these.

The included studies were conducted among the non-institutionalised and non-hospitalised population. Especially severe tinnitus showed a strong association with general morbidity and psychiatric disorders. ${ }^{58}$ Therefore, selection bias may have affected the results. One of the included studies was conducted among operating airline pilots, which are for safety reasons not allowed to work when suffering from severe tinnitus. ${ }^{65}$ Another survey was performed among students of a certain region, excluding those who visited special schools for adolescents with audiological or general disabilities. ${ }^{69}$ Thus, it is likely that severe tinnitus cases are under-represented within the included studies. The results of this analysis support the assumption that smoking is predominantly associated with severe tinnitus. In that way, the dropout of tinnitus cases is not equally distributed among smokers and non-smokers, which could attenuate the observed association when general tinnitus is assessed.

Misclassification of exposure and outcome variables may have biased the results towards a null effect. Some studies assessed smoking as a dichotomous variable, 
not indicating whether ex-smokers were assigned to the smoking or non-smoking group or excluded from the analysis. ${ }^{67} 89$ Even if smoking classification was assessed in terms of current, former or never smoking, the definitions of each category differed across studies or were not provided at all. Furthermore, the effect of passive smoking exposure was taken into account by one study only. ${ }^{85}$

Concerning the quality assessment of studies, the tool applied was neither checked for validity nor reliability. However, none of the validated tools deemed appropriate, as items that seemed vital in the context of this review were not included. Besides a focus on quality and low risk of bias, the questions of comparability played an essential role in the selection of a less heterogeneous subgroup.

Another limitation of this meta-analysis is that most of the included studies correspond to a cross-sectional study design. Therefore, the observed association does not allow conclusions about causality. An approach in addressing the question of causality is the investigation of dose-response relationships. Hoffman and Reed examined the prevalence of tinnitus depending on the number of years participants had smoked daily, without illustrating a clear dose-response relationship. ${ }^{47}$ Similarly, Davis $e t a b^{67}$ did not observe a correlation between tinnitus and quantity smoked. Nondahl et at ${ }^{45}$ conducted similar analyses, yet providing some vague evidence of a dose-response trend. Another approach to providing evidence of a causal relationship is longitudinal research. Five cohort studies could be included in the different comparisons. Regarding former and ever smoking, the pooled risks of cohort studies are more pronounced than those of cross-sectional studies, whereas regarding current smoking, cohort studies indicated a somewhat smaller risk than cross-sectional studies.

Finally, the weights of individual studies were obtained by inverse variance, which correlates most often with sample size. Whereas the impact of bias in randomised controlled trials can be reduced by an increase of sample size, this is not transferable to observational studies. ${ }^{90} \mathrm{In}$ this way, instead of reducing the effect of bias, a meta-analysis of observational studies can even boost the impact of bias. ${ }^{90}$ Meta-analyses that pool primary data instead of approximated effect sizes may be preferable. ${ }^{91}$ However, as a first investigation on this topic, this method deemed appropriate.

\section{CONCLUSION}

The results of this meta-analysis show a statistically significant association between smoking and tinnitus. However, this association does not provide proof of a causal relationship. A number of interfering variables may have masked the level of this association to some extent. In order to draw more precise conclusions, further evidence is needed. As pointed out by several authors, the agreement on a universal definition of pathologic tinnitus is of fundamental importance to gain new evidence. ${ }^{92} 93$ So far, the definition applied most often is the one of prolonged spontaneous tinnitus which defines tinnitus lasting longer than $5 \mathrm{~min}$ and not occurring in sequence to strong noise exposure. ${ }^{92} 94$ A clear categorisation of smoking status, for example, in terms of current, former and never-smoker is equally needed to facilitate comparability across studies. Moreover, longitudinal studies and contemplation of tinnitus in dependence of pack years are crucial approaches to elucidate questions of causality. Another emphasis has to be put on the importance of adjustments for other variables such as noise exposure, stress and stress coping skills, psychological comorbidity and cardiovascular risk factors. People suffering from tinnitus should be educated about the potential impact of smoking. Especially in noise-exposed populations, education and smoking cessation campaigns are a worthwhile approach.

Acknowledgements The authors sincerely thank Annette Kifley, Paul Mitchell and Bamini Gopinath for contributing additional data from the Blue Mountain Hearing Study (University of Sydney). The authors equally thank Goran Belojevic (University of Belgrade) for providing data to the analysis.

Contributors AV was involved in design and conception of the work. She performed the systematic literature search, set up in and exclusion criteria in collaboration with the other coauthors. She designed a tool for study quality assessment, ran the statistical analyses and drafted the manuscript. She is responsible for the overall content as a guarantor. $\mathrm{HZ}$ performed an independent systematic literature search as a second reviewer on the topic. He was involved in conception and design of the work. EL gave substantial methodological input. She critically revised some of the calculations, and several versions of the manuscript. $\mathrm{HB}$ lanced the project. As a supervisor, he directed and mentored every step of the review such as design and conception, statistical analyses, questions of inclusion and exclusion criteria. He is responsible for the overall content as a guarantor. All authors contributed to design of the work, interpretation of data and revising drafts. All authors approved the final version of the manuscript and agreed to be accountable for the aspects of their contributions.

Funding We acknowledge financial support by Deutsche Forschungsgemeinschaft within the funding programme Open Access Publishing, by the Baden-Württemberg Ministry of Science, Research and the Arts and by Ruprecht-Karls-Universität Heidelberg.

Competing interests None declared.

Patient consent Not required.

Provenance and peer review Not commissioned; externally peer reviewed.

Data sharing statement Additional data is available from the corresponding author upon request (h.becher@uke.de).

Open Access This is an Open Access article distributed in accordance with the Creative Commons Attribution Non Commercial (CC BY-NC 4.0) license, which permits others to distribute, remix, adapt, build upon this work non-commercially, and license their derivative works on different terms, provided the original work is properly cited and the use is non-commercial. See: http://creativecommons.org/ licenses/by-nc/4.0/

(c) Article author(s) (or their employer(s) unless otherwise stated in the text of the article) 2018. All rights reserved. No commercial use is permitted unless otherwise expressly granted.

\section{REFERENCES}

1. Kompis M, Neuner NT, Hemmeler W, et al. [Tinnitus]. Ther Umsch 2004;61:15-20.

2. Degeest $\mathrm{S}$, Keppler $\mathrm{H}$, Corthals $\mathrm{P}$, et al. Epidemiology and risk factors for tinnitus after leisure noise exposure in Flemish young adults. Int J Audiol 2017;56:121-9. 
3. Hébert S, Carrier J. Sleep complaints in elderly tinnitus patients: a controlled study. Ear Hear 2007;28:649-55.

4. Holgers KM, Erlandsson SI, Barrenäs ML. Predictive factors for the severity of tinnitus. Audiology 2000;39:284-91.

5. Mohamad N, Hoare DJ, Hall DA. The consequences of tinnitus and tinnitus severity on cognition: A review of the behavioural evidence. Hear Res 2016;332:199-209.

6. Nondahl DM, Cruickshanks KJ, Wiley TL, et al. Prevalence and 5 -year incidence of tinnitus among older adults: the epidemiology of hearing loss study. J Am Acad Audiol 2002;13:323-31.

7. Palmer KT, Griffin MJ, Syddall HE, et al. Cigarette smoking, occupational exposure to noise, and self reported hearing difficulties. Occup Environ Med 2004;61:340-4.

8. Zenner H-P. Eine Systematik für Entstehungsmechanismen von Tinnitus. HNO 1998:46:699-704.

9. Henry JA, Dennis KC, Schechter MA. General review of tinnitus: prevalence, mechanisms, effects, and management. J Speech Lang Hear Res 2005;48:1204-35.

10. Mazurek B, Haupt $\mathrm{H}$, Georgiewa $\mathrm{P}$, et al. A model of peripherally developing hearing loss and tinnitus based on the role of hypoxia and ischemia. Med Hypotheses 2006;67:892-9.

11. Frost-Pineda $\mathrm{K}$, Liang $\mathrm{Q}$, Liu J, et al. Biomarkers of potential harm among adult smokers and nonsmokers in the total exposure study. Nicotine Tob Res 2011;13:182-93.

12. Al Rifai M, DeFilippis AP, McEvoy JW, et al. The relationship between smoking intensity and subclinical cardiovascular injury: The Multi-Ethnic Study of Atherosclerosis (MESA). Atherosclerosis 2017;258:119-30.

13. Yanbaeva DG, Dentener MA, Creutzberg EC, et al. Systemic effects of smoking. Chest 2007;131:1557-66.

14. Messner B, Bernhard D. Smoking and cardiovascular disease: mechanisms of endothelial dysfunction and early atherogenesis. Arterioscler Thromb Vasc Biol 2014;34:509-15.

15. Turner JA, McNicol MW, Sillett RW. Distribution of carboxyhaemoglobin concentrations in smokers and non-smokers. Thorax 1986;41:25-7.

16. Walther LE, Hülse R, Lauer $\mathrm{K}$, et al. [Current aspects of ototoxicity. Ototoxic substances and their effects]. HNO 2015;63:315-24; quiz 325-6.

17. Talhout R, Schulz T, Florek E, et al. Hazardous compounds in tobacco smoke. Int J Environ Res Public Health 2011;8:613-28.

18. Harkrider AW, Champlin CA, McFadden D. Acute effect of nicotine on non-smokers: I. OAEs and ABRs. Hear Res 2001:160(1-2):73-88.

19. Pomerleau OF, Rosecrans J. Neuroregulatory effects of nicotine. Psychoneuroendocrinology 1989;14:407-23.

20. Dani JA, Ji D, Zhou FM. Synaptic plasticity and nicotine addiction. Neuron 2001;31:349-52.

21. Kirk KM, McGuire A, Nielsen L, et al. Self-reported tinnitus and ototoxic exposures among deployed Australian Defence Force personnel. Mil Med 2011;176:461-7.

22. Stouffer JL, Tyler RS. Characterization of tinnitus by tinnitus patients. $J$ Speech Hear Disord 1990;55:439-53.

23. Leitlinien AdWMFA-SK. AWMF- S3-Leitlinie 017/064: Chronischer Tinnitus: Auflage, 2015. http://www.awmf.org/uploads/tx_szleitlinien

24. Tunkel DE, Bauer CA, Sun GH, et al. Clinical practice guideline: tinnitus. Otolaryngol Head Neck Surg 2014;151(2 Suppl):S1-S40.

25. Liberati A, Altman DG, Tetzlaff $\mathrm{J}$, et al. The PRISMA statement for reporting systematic reviews and meta-analyses of studies that evaluate health care interventions: explanation and elaboration. PLOS Med 2009;6:e1000100.

26. Stroup DF, Berlin JA, Morton SC, et al. Meta-analysis of observational studies in epidemiology: A proposal for reporting. JAMA 2000;283:2008-12.

27. Higgins JPT, Green S. Cochrane Handbook for Systematic Reviews of Interventions: The Cochrane Collaboration, 2011. Version 5.1.0.

28. Hazell J. Proceedings of the Sixth International Tinnitus Seminar 1999. Special Edition. Cambridge UK: Hosted by the British Society of Audiology, 2002.

29. Patuzzi RH. Proceedings of the Seventh International Tinnitus Seminar. Perth: University of Western Australia, 2002.

30. Reich GE VJH. Proceedings of the Fifth International Tinnitus Seminar. Portland: American Tinnitus Association, 1996.

31. Reh DD, Higgins TS, Smith TL. Impact of tobacco smoke on chronic rhinosinusitis: a review of the literature. Int Forum Allergy Rhino 2012;2:362-9.

32. Hur K, Liang J, Lin SY. The role of secondhand smoke in sinusitis: a systematic review. Int Forum Allergy Rhinol 2014;4:22-8.

33. Lambert R, Sauvaget C, de Camargo Cancela M, et al. Epidemiology of cancer from the oral cavity and oropharynx. Eur J Gastroenterol Hepatol 2011;23:633-41.
34. Huber MA, Tantiwongkosi B. Oral and oropharyngeal cancer. Med Clin North Am 2014;98:1299-321.

35. Kong K, Coates HL. Natural history, definitions, risk factors and burden of otitis media. Med J Aust 2009;191(9 Suppl):S39-43.

36. Jones LL, Hassanien A, Cook DG, et al. Parental smoking and the risk of middle ear disease in children: a systematic review and metaanalysis. Arch Pediatr Adolesc Med 2012;166:18-27.

37. Nomura K, Nakao M, Morimoto T. Effect of smoking on hearing loss: quality assessment and meta-analysis. Prev Med 2005;40:138-44.

38. Jüni $P$, Witschi $A$, Bloch $R$, et al. The hazards of scoring the quality of clinical trials for meta-analysis. JAMA 1999;282:1054-60.

39. Downs $\mathrm{SH}$, Black N. The feasibility of creating a checklist for the assessment of the methodological quality both of randomised and non-randomised studies of health care interventions. J Epidemiol Community Health 1998:52:377-84.

40. Sanderson S, Tatt ID, Higgins JP. Tools for assessing quality and susceptibility to bias in observational studies in epidemiology: a systematic review and annotated bibliography. Int J Epidemiol 2007;36:666-76

41. Shamliyan T, Kane RL, Dickinson S. A systematic review of tools used to assess the quality of observational studies that examine incidence or prevalence and risk factors for diseases. J Clin Epidemiol 2010;63:1061-70.

42 von Elm E, Altman DG, Egger M, et al. STROBE Initiative. The strengthening the reporting of observational studies in epidemiology (STROBE) statement: guidelines for reporting observational studies. Prev Med 2007;45:247-51.

43. Borenstein M, ed. Introduction to meta-analysis. Repr. ed. Chichester: Wiley, 2010.

44. Fujii K, Nagata C, Nakamura K, et al. Prevalence of tinnitus in community-dwelling Japanese adults. J Epidemiol 2011;21:299-304

45. Nondahl DM, Cruickshanks KJ, Huang GH, et al. Tinnitus and its risk factors in the Beaver Dam offspring study. Int J Audiol 2011;50:313-20.

46. Nondahl DM, Cruickshanks KJ, Wiley TL, et al. The ten-year incidence of tinnitus among older adults. Int J Audiol 2010;49:580-5.

47. Hoffman HJ, Reed GW. Epidemiology of tinnitus. Tinnitus: Theory and management. Snow JB Jr, ed. Tinnitus: Theory and Management. Hamilton, Ontario: B.C. Decker, Inc, 2004:16-41.

48. Kim HJ, Lee HJ, An SY, et al. Analysis of the prevalence and associated risk factors of tinnitus in adults. PLoS One 2015;10:e0127578

49. McCormack A, Edmondson-Jones M, Mellor D, et al. Association of Dietary Factors with Presence and Severity of Tinnitus in a Middle-Aged UK Population (vol 9, e114711, 2014). Plos One 2015;10:1

50. Altman DG, Bland JM. Interaction revisited: the difference between two estimates. BMJ 2003;326:219.

51. Park KH, Lee SH, Koo JW, et al. Prevalence and associated factors of tinnitus: data from the Korean National Health and Nutrition Examination Survey 2009-2011. J Epidemiol 2014;24:417-26.

52. Park RJ, Moon JD. Prevalence and risk factors of tinnitus: the Korean National Health and Nutrition Examination Survey 2010-2011, a cross-sectional study. Clin Otolaryngol 2014;39:89-94.

53. Seo JH, Kang JM, Hwang SH, et al. Relationship between tinnitus and suicidal behaviour in Korean men and women: a cross-sectional study. Clin Otolaryngol 2016;41.

54. Hébert S, Canlon B, Hasson D. Emotional exhaustion as a predictor of tinnitus. Psychother Psychosom 2012;81:324-6.

55. Joo YH, Han KD, Park KH. Association of hearing loss and tinnitus with health-related quality of life: The korea national health and nutrition examination survey. PLoS One 2015;10:e0131247.

56. Sogebi OA. Characterization of tinnitus in Nigeria. Auris Nasus Larynx 2013;40:356-60

57. Martines F, Sireci F, Cannizzaro E, et al. Clinical observations and risk factors for tinnitus in a Sicilian cohort. Eur Arch Otorhinolaryngol 2015;272:2719-29.

58. Holgers KM, Zöger S, Svedlund K. Predictive factors for development of severe tinnitus suffering-further characterisation. Int $J$ Audiol 2005:44:584-92.

59. Rubak T, Kock S, Koefoed-Nielsen B, et al. The risk of tinnitus following occupational noise exposure in workers with hearing loss or normal hearing. Int J Audiol 2008;47:109-14.

60. Martinez C, Wallenhorst C, McFerran D, et al. Incidence rates of clinically significant tinnitus: 10-year trend from a cohort study in England. Ear Hear 2015;36:e69-75.

61. Paschoal CP, Azevedo MF. Cigarette smoking as a risk factor for auditory problems. Braz J Otorhinolaryngol 2009;75:893-902.

62. Palmer KT, Griffin MJ, Syddall HE, et al. Occupational exposure to noise and the attributable burden of hearing difficulties in Great Britain. Occup Environ Med 2002;59:634-9. 
63. Sindhusake D, Golding M, Newall P, et al. Risk factors for tinnitus in a population of older adults: the blue mountains hearing study. Ear Hear 2003;24:501-7.

64. Sindhusake D, Golding M, Wigney D, et al. Factors predicting severity of tinnitus: a population-based assessment. J Am Acad Audiol 2004;15:269-80.

65. Lindgren T, Wieslander G, Dammström BG, et al. Tinnitus among airline pilots: prevalence and effects of age, flight experience, and other noise. Aviat Space Environ Med 2009;80:112-6.

66. Lasisi AO, Abiona T, Gureje O. Tinnitus in the elderly: Profile, correlates, and impact in the Nigerian Study of Ageing. Otolaryngol Head Neck Surg 2010;143:510-5.

67. Davis AC C, Smith PA, Spencer HS. Factors influencing tinnitus report in Great Britain. In: Aran J-M, Dauman R, Proceedings of the Fourth International tinnitus Seminar Bordeaux. 1991. Amsterdam/ New York: Kugler Publications, 1992:239-43.

68. Marmut Z, Belojevic G, Backovic D, et al. Tinnitus among Serbian secondary school students in relation to their behavior and habits. Noise Health. Mar 2014;16:73-8.

69. Brunnberg $E$, Lindén-Boström $M$, Berglund $M$. Tinnitus and hearing loss in 15-16-year-old students: Mental health symptoms, substance use, and exposure in school. Int J Audiol 2008;47:688-94.

70. Egger M, ed. Public Health: Sozial- und Präventivmedizin kompakt. 2., aktual. Aufl. ed. Berlin [u.a.]: De Gruyter: Studium, 2014.

71. Gesundheitswesen - Fragen zur Gesundheit - Rauchgewohnheiten der Bevölkerung, Mikrozensus 20130 Statistisches Bundesamt, Wiesbaden 2014: Artikelnummer: 5239004139004, 2014. https:// www.destatis.de/DE/Publikationen/ Thematisch/Gesundheit/ Gesundheitszustand/Rauchgewohnheiten.html (accessed 28 Sep 2015).

72. Gallus S, Lugo A, Garavello W, et al. Prevalence and determinants of tinnitus in the italian adult population. Neuroepidemiology 2015;45:12-19.

73. Michikawa T, Nishiwaki Y, Kikuchi Y, et al. Prevalence and factors associated with tinnitus: a community-based study of Japanese elders. J Epidemiol 2010;20:271-6.

74. Bohlin M, Erlandsson S. Risk behaviour and noise exposure among adolescents. Noise and Health 2007;9:55-63.

75. Nondahl DM, Cruickshanks KJ, Dalton DS, et al. The use of hearing protection devices by older adults during recreational noise exposure. Noise and Health 2006;8:147-53.

76. Larson NI, Story M, Neumark-Sztainer D, et al. are diet and physical activity patterns related to cigarette smoking in adolescents? findings from project EAT. Preventing Chronic Disease 2007;4:A51.

77. Härter M, Maurischat C, Weske G, et al. Psychological stress and impaired quality of life in patients with tinnitus]. HNO 2004;52:125-31.
78. Geocze L, Mucci S, Abranches DC, et al. Systematic review on the evidences of an association between tinnitus and depression. Braz J Otorhinolaryngol 2013;79:106-11.

79. Morissette SB, Tull MT, Gulliver SB, et al. Anxiety, anxiety disorders, tobacco use, and nicotine: A critical review of interrelationships. Psychol Bull 2007;133:245-72.

80. Trosclair A, Dube SR. Smoking among adults reporting lifetime depression, anxiety, anxiety with depression, and major depressive episode, United States, 2005-2006. Addict Behav 2010;35:438-43.

81. D'Amelio R, Archonti C, Scholz S, et al. Psychological distress associated with acute tinnitus]. HNO 2004;52:599-603.

82. Welch D, Dawes PJD. Personality and perception of tinnitus. Ear Hear 2008;29:684-92.

83. Cui $X$, Rockett IRH, Yang T, et al. Work stress, life stress, and smoking among rural-urban migrant workers in China. BMC Public Health 2012;12:979.

84. Chung DY, Gannon RP, Mason K. Factors affecting the prevalence of tinnitus. Audiology 1984;23:441-52.

85. Mahboubi H, Oliaei S, Kiumehr S, et al. The prevalence and characteristics of tinnitus in the youth population of the United States. Laryngoscope 2013;123:2001-8.

86. Shargorodsky J, Curhan GC, Farwell WR. Prevalence and characteristics of tinnitus among US adults. Am J Med 2010;123:711-8.

87. Dwan K, Gamble C, Williamson PR, et al. Systematic review of the empirical evidence of study publication bias and outcome reporting bias - an updated review. PLoS One 2013;8:e66844.

88. Holgers KM, Pettersson B. Noise exposure and subjective hearing symptoms among school children in Sweden. Noise and Health 2005;7:27-37.

89. Canlon B, Theorell T, Hasson D. Associations between stress and hearing problems in humans. Hear Res 2013;295:9-15.

90. Blettner M, Schlehofer B, Sauerbrei W. Grenzen von Metaanalysen aus publizierten Daten bei epidemiologischen Fragestellungen. Sozial- und Präventivmedizin. SPM 1997;42:95-104.

91. Chang BH, Hoaglin DC. Meta-analysis of OR: current good practices. Med Care 2017;55:328-35.

92. McCormack A, Edmondson-Jones M, Somerset S, et al. A systematic review of the reporting of tinnitus prevalence and severity. Hear Res 2016;337:70-9.

93. Sanchez L. The epidemiology of tinnitus. Audiol Med 2004;2:8-17.

94. Coles R. Aetiology and classification. In: Reich GE, Vernon JA, eds. Proceedings of the Fifth International Tinnitus Seminar 95 Portland, OR: American Tinnitus Association, Epidemiology, 1996:25-9. 